Case Report

\title{
Florid Cemento-Osseous Dysplasia of Osteolytic Stage Showing Cyst-Like Findings on CT and MRI: A Case Report
}

\author{
Kotaro Ito, ${ }^{1}$ Naohisa Hirahara, ${ }^{1}$ Norihito Iizuka, ${ }^{2}$ Eri Sawada, ${ }^{2}$ Shunya Okada, ${ }^{2}$ Masaaki Suemitsu, ${ }^{3}$ \\ Kayo Kuyama, ${ }^{3}$ and Takashi Kaneda ${ }^{1}$ \\ Departments of ${ }^{1}$ Radiology and ${ }^{3}$ Pathology, Nihon University School of Dentistry at Matsudo, Matsudo, Chiba 271-8587, Japan \\ ${ }^{2}$ Nihon University Graduate School of Dentistry at Matsudo, Radiology, Matsudo, Chiba 271-8587, Japan
}

\section{Article History}

Received 30 November 2018

Accepted 18 December 2018
Keywords :

florid cement-osseous dysplasia,

periapical cemental dysplasia,

fibro- osseous lesion,

magnetic resonance imaging

\begin{abstract}
We report our experience of florid cemento-osseous dysplasia(FCOD) showing cyst-like findings on Computed tomography (CT) and Magnetic resonance imaging (MRI), along with some literature review. A 36-year-old Japanese woman was referred to department of Oral and Maxillofacial surgery, Nihon University School of Dentistry at Matsudo for a radiolucent lesion in lower right molar area. Pantomogram showed multiple cystic lesions of the mandible. CT demonstrated the well-defined multilocular homogeneous waterdensity lesions in right molar, incisor and left molar areas. No obvious the buccolingual bone expansion and displacement of the inferior alveolar canal due to the lesions were observed. T1-weighted MRI showed low signal intensity lesions. T2-weighted MRI showed heterogeneous high signal intensity lesions. Postcontrast T1-weighted MRI showed low signal intensity lesions with peripheral contrast-enhancement like cystic lesion. These findings led to a suspicion of cystic lesions, and we performed cystectomy. Histopathologically, cellular fibrous stroma with trabecular bone and cementum-like materials were shown. There was no arrangement of osteoblasts around the bony tissue. Dilated blood vessels were also observed in the stroma. The diagnosis of FCOD was established based on the histopathological examination combined with the imaging findings. The postoperative course over two years were uneventful.
\end{abstract}

\section{Introduction}

Cemento-Osseous Dysplasia (COD) is the most common fibro-osseous lesion of the jaws affecting wide range of the maxilla and mandible. $(1,2)$. COD was classified as benign odontogenic neoplasms of the jaws in the 2005 World Health Organization (WHO) classification, updated in 2017 (2, 3). Whereas historically, COD was thought to be cementoosseous, in 2001 Fowler and Brannon reported that this lesion was composed of bone and not cementum (4). However, it was difficult to distinguish between cementumlike structure and abnormal bone pathologically, so the name was changed to COD again in the 2017 WHO classification(3). COD was classified as periapical cemental dysplasia and florid cemento-osseous dysplasia (FCOD)

Correspondence to :

Kotaro Ito

E-mail : itou.koutarou@nihon-u.ac.jp depending on clinic-radiographic. Extensive from of COD affecting three or more quadrants or widespread involvement in a jaw is defined as $\operatorname{FCOD}(1,5)$. FCOD is the most extensive fibro- osseous lesion involving multiple quadrants of the jaws. The etiopathogenesis of FCOD is unknown but could occur from the medullar bone, the periodontal ligament or both(6).

COD is a lesion of histologically benign in which normal bone is replaced by fibro- osseous tissue. Histopathological analysis of COD revealed a cellular fibrous connective tissue including cementum-like structure and abnormal bone, accumulating over time $(2,7)$. COD mature and become larger and more radiopaque with time. COD is classified into the three stages based on radiological findings: 1) osteolytic stage, 2) mixed stage and 3) osteogenic stage (5, 6, 8).

Because of most FCOD are asymptomatic, FCOD is usually found by routine dental examinations, especially in 


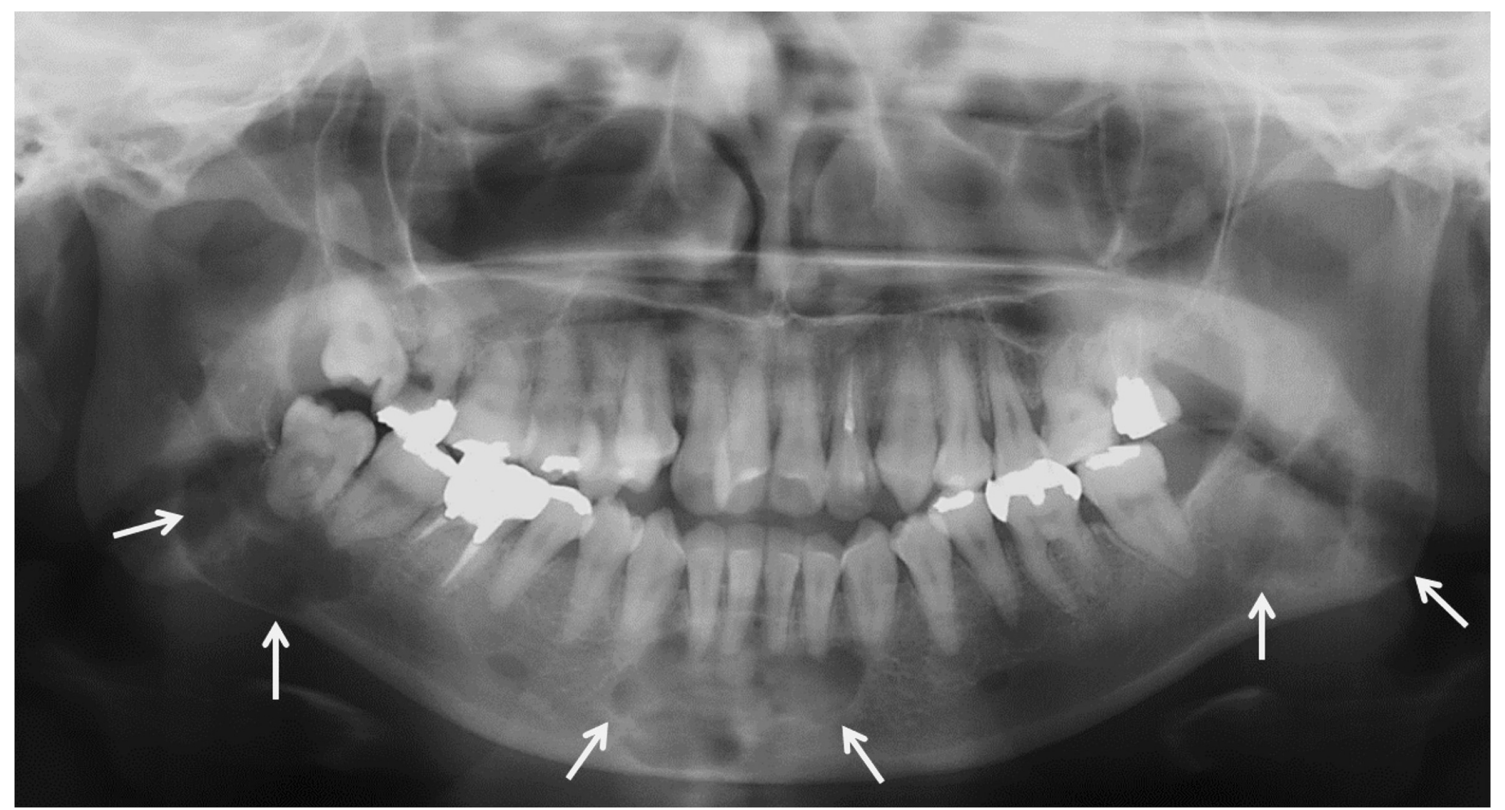

Fig. 1. Pantomogram shows multiple cystic lesions of the mandible(Arrows). These lesions have well-defined multilocular radiolucent appearance.

4th-5th decades female commonly in black and Asians (1, 2, 9-11). Diagnosis of FCOD is based on the typical radiographic appearance assessed with standard twodimensional projection radiographs (intraoral radiography or Pantomogram). In atypical cases, three-dimensional radiographic assessment using Computed tomography (CT) is mandatory, especially in FCOD of osteolytic stage, to facilitate differential diagnosis with other radiolucent lesions (2).

Biopsy of FCOD is not recommended in typical presentations because of the risk of significant postoperative infection, probably secondary to the reduced vascular supply in these lesions $(1,12)$. Therefore, differential diagnosis of FCOD and other lesions is very important. However, it is often difficult to differentiation between FCOD and other lesions because FCOD shows various imaging findings depending on lesion stage. Furthermore, there are few reports of Magnetic resonance imaging(MRI) findings of FCOD of osteolytic stage.

In this study, we report our experience of FCOD showing cystic-like findings on CT and MRI, along with some literature review.

\section{Case report}

In 2014, A 36-year-old Japanese woman presented to primary dental clinic with pain of lower right molar area and was noted have a radiolucent lesion in lower right molar area. She was referred to department of Oral and Maxillofacial surgery, Nihon University School of Dentistry at Matsudo for further examination and treatment. She had improved her symptoms when she presented our hospital, because she was prescribed nonsteroidal anti-inflammatory medications from previous doctor.

Her past medical history was not notable, and there was no evidence of systemic disease. Also, her family history was unremarkable. On intraoral examination, the patient was asymptomatic, there was no swelling of the oral mucosa and all present teeth were vital except the upper left lateral incisor, the first lower right molar and the third upper left molar. Pantomogram and CT examinations were performed in oral and maxillofacial area (Fig. 1,2).

Pantomogram showed multiple cystic lesions of the mandible. In the mandible, the apical area of the second and third right molar, apical area of incisors and the edentulous area of the left third molar were involved. In right molar area, the lesion extended from the root of the second right molar to distal root of the third molar and from the root of second and third molar to the inferior alveolar canal. The lesion had well-defined multilocular radiolucent appearance. Displacement of the inferior alveolar canal due to the lesion was not observed. In incisors area, the lesion extended mesiodistally from the root of left lateral incisor to the root 

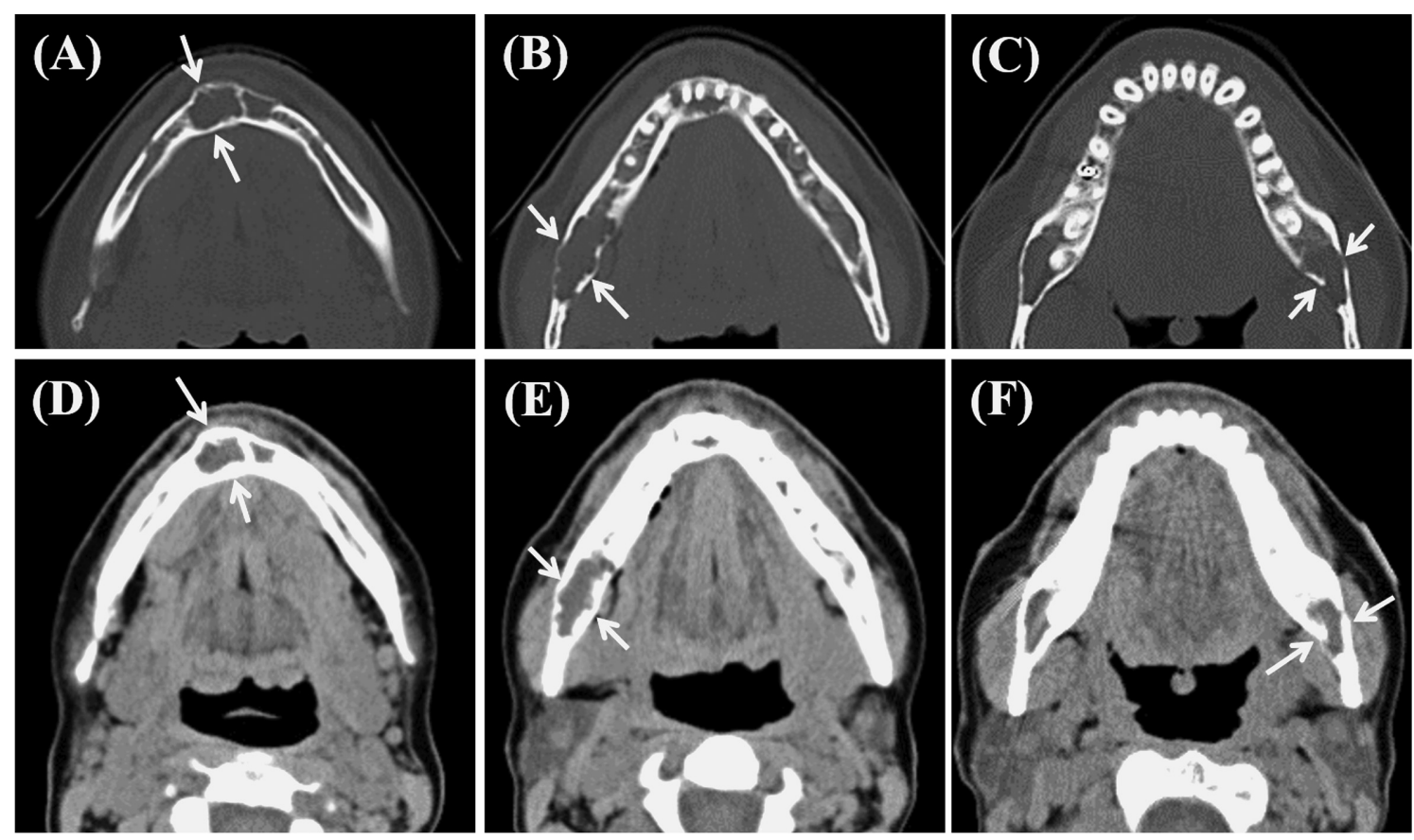

Fig. 2. Axial CT images show the well-defined multilocular homogeneous water-density lesions in right molar incisor and left molar areas (Arrows). The lesions have the same density as water, and there are no obvious buccolingual bone expansion of the jawbone.

of right lateral incisor. The lesion had well-defined multilocular radiolucent appearance. In edentulous area of the left third molar, the lesion had well-defined multilocular radiolucent appearance. Displacement of the inferior alveolar canal due to the lesion was not observed. In addition, Pantomogram showed well-defined unilocular round radiolucent lesions of right second molar, left first and second molar areas. In all lesion areas, there were no root resorption and no displacement of the involved teeth. Also, localized periodontal bone loss was observed in the alveolar bone of distal of the lower third right molar.

CT images showed the well-defined multilocular homogeneous water-density lesions in right molar, incisor and left molar areas. Axial CT images showed the no obvious buccolingual bone expansion in all area. Based on these findings, the patient was diagnosed jaw cysts temporarily.

For differential diagnosis, MRI examinations with and without contrast-enhanced were performed as additional examinations (Fig. 3). Axial T1-weighted MRI showed low signal intensity lesions. Axial T2-weighted MRI showed heterogeneous high signal intensity lesions. Axial postcontrast T1-weighted MRI showed low signal intensity lesions with peripheral contrast-enhancement like cystic lesion.

These findings led to a suspicion of cystic lesions, and we performed cystectomy. Histopathology showed cellular fibrous stroma with trabecular bone and cementum-like tissues. No arrangement of osteoblasts or cementoblasts was observed at the periphery of the hard tissues. Dilates blood vessels were also identified in the fibrous stroma (Fig. 4). The diagnosis of FCOD was established based on the histopathological examination combined with the radiographic findings. The postoperative course over two years were uneventful.

\section{Discussion}

FCOD occurs frequently in middle-aged Africans and Asian women (1, 2, 10-12). The reason for this gender and racial predilection and the etiology of FCOD are unknown but this lesion is thought to occur from periodontal ligament $(5,13)$. Also, in the past literature reported that hormone imbalance affects the occurrence of FCOD (14). Some cases have been reported to show a family history, but most cases appear to represent isolated instances $(10,11,12)$. However, since gender, race, and age are important factors for the diagnosis of NPDC, they must be considered to add FCOD for differentiation when lesions are observed in middle-aged Asian women, as in this case. Generally, FCOD is asymptomatic $(1,2)$. The patient had pain in this case, 

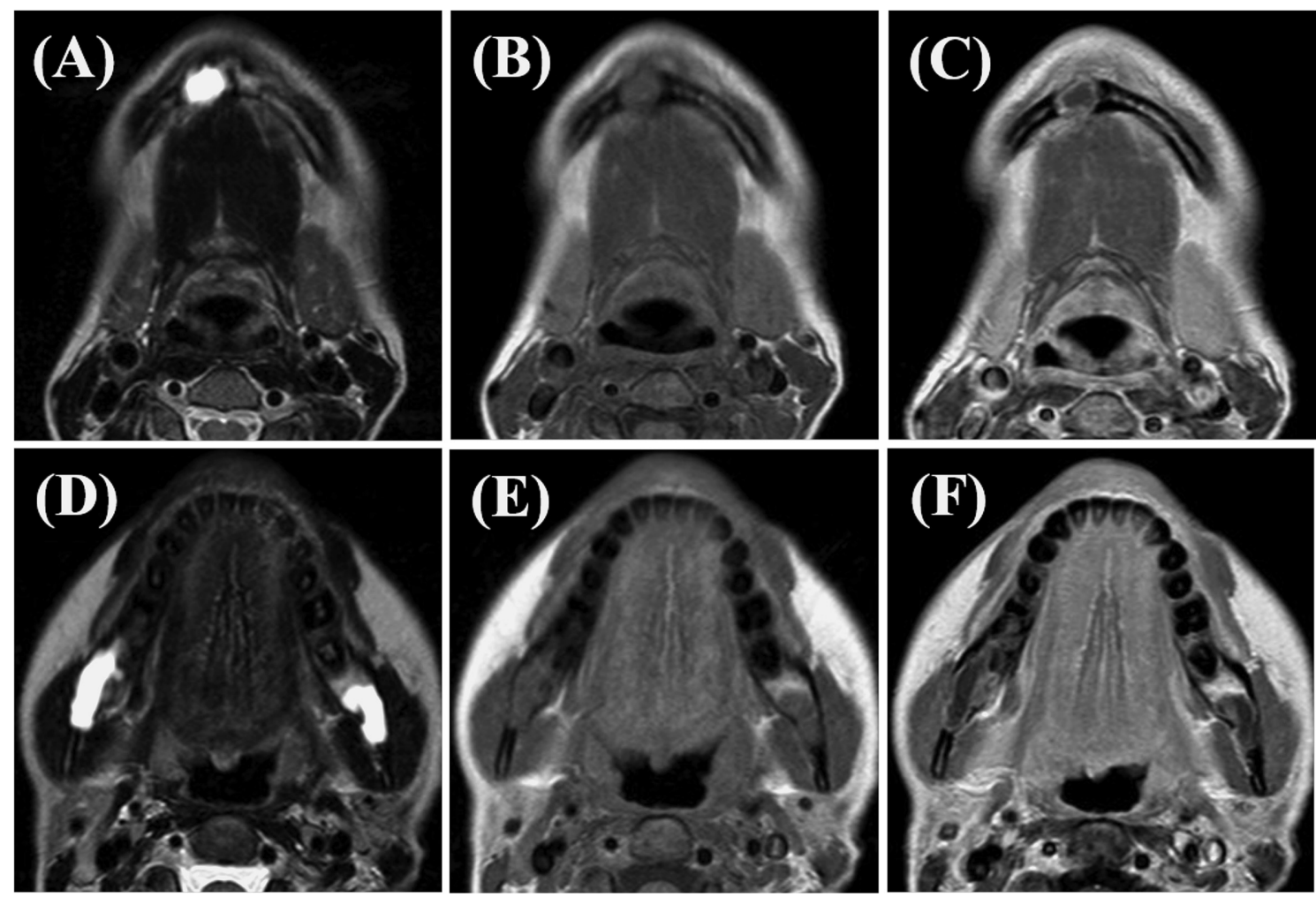

Fig. 3. Axial MR images show FCOD of osteolytic stage. (A, D) T2-weighted shows heterogeneous high signal intensity lesions. (B, E) T1-weighted MRI shows low signal intensity lesions. (C, F) postcontrast T1weighted MRI showed low signal intensity lesions with peripheral contrast-enhancement like cystic lesions.

however, there were no findings suspected of inflammation from image findings and pathological findings. Also, since symptoms improved after prescription nonsteroidal antiinflammatory medications, the pain was thought to be due to pericoronitis of lower third right molar.

FCOD can be differentiated from other bony lesions such as Paget's disease and, fibrous dysplasia, as well as Osteomyelitis $(1,15)$. FCOD of mixed and osteogenic stage shows the "cotton-wool appearance" and "ground-glass appearance" aspect which are also typical of Paget's diseases and fibrous dysplasia (1, 16). Also, FCOD with infection shows sequestra and wider radiolucent border findings like osteomyelitis. Secondary infection can occasionally occur in FCOD, and its management is known to be complicated (17). The infection is possibly secondary to osteomyelitis in an adjacent bone, or it is possible that FCOD makes the bone more susceptible to osteomyelitis $(18,19)$. However, in this case, the FCOD without infection was osteolytic stage and did not show these findings. Therefore, these diseases were not differentiated. In addition, when FCOD of osteolytic stage occurs on a tooth after root canal treatment, apical periodontitis and radicular cysts can be differentiate(1). Since all teeth including FCOD were vital in this case, these diseases were excluded from the diagnosis. For multiple cystic lesions of the jaw, basal cell nevus syndrome, cherubism and Langerhans cell histiocytosis are considered (20). However, these diseases occur frequently in young ages (20-22). Also, basal cell nevus syndrome and cherubism show the findings of buccolingual bone expansion $(20,21)$. Langerhans cell histiocytosis is lesion affecting reticuloendothelial system and characterized by proliferation of Langerhans cells, and it shows the findings of "floating teeth" and "scooped out" appearances (22). These findings were not consistent in this case.

In this case, FCOD showed cyst-like findings on MRI such as well-defined margin, low T1 high T2 signal intensity of MRI and low signal intensity lesions with peripheral contrast-enhancement of postcontrast T1-weighted MRI. These were consistent with typical cystic lesions findings. For these reasons, cystic lesions were most suspected in this case. There are many reports that FCOD of osteolytic stage shows X-ray permeability, but there are few reports of MRI 


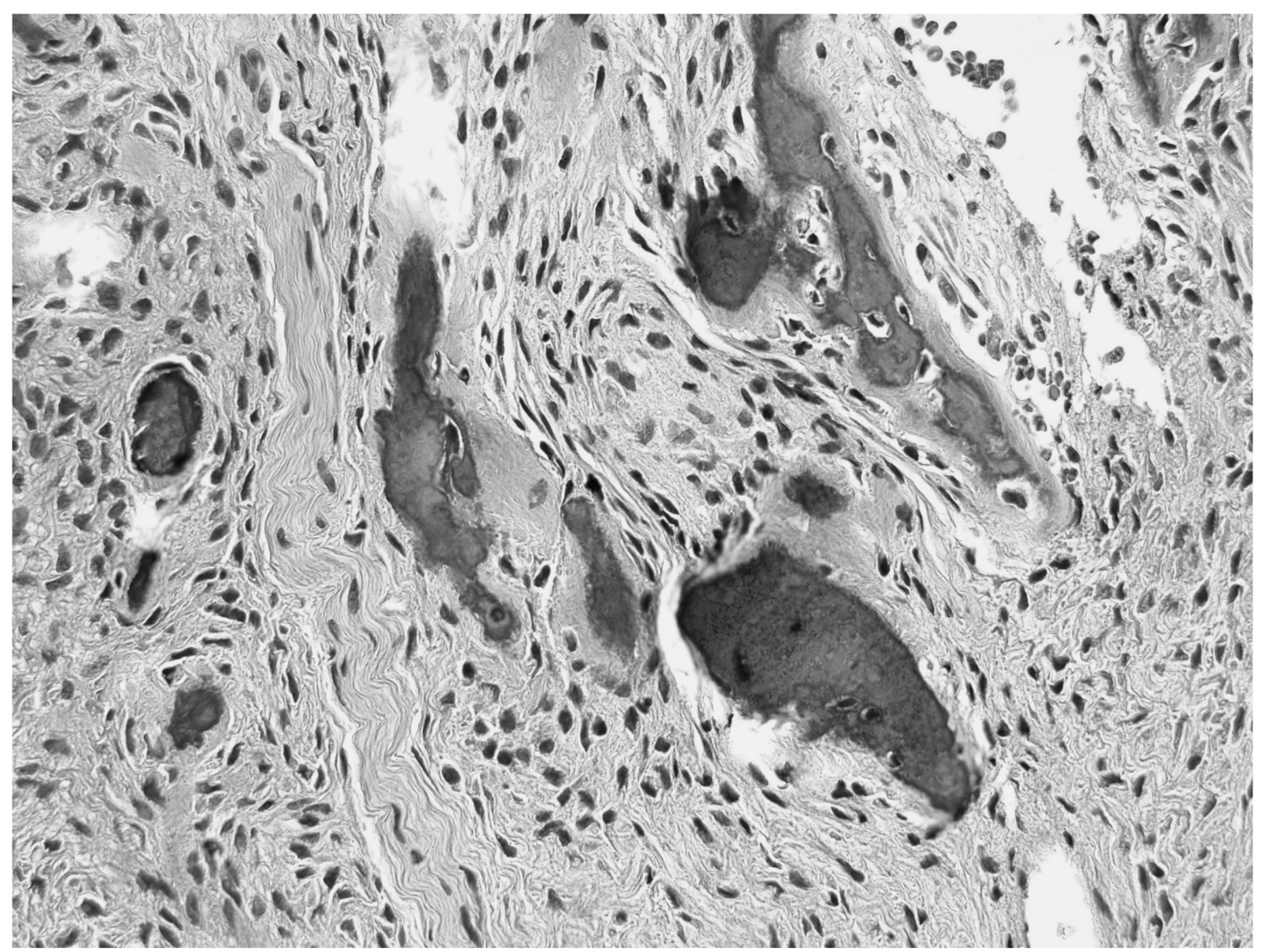

Fig. 4. Histopathological findings of osteolytic state of FCOD $(40 \times$ magnification $)$ Cellular fibrous stroma and scattered bone and cementum-like hard tissue fragments were evident. No osteoblastic and cementoblastic arrangements were observed at the periphery of the hard tissue matrices.

findings of FCOD of osteolytic stage $(1,2,16)$. This case elucidated that FCOD of osteolytic stage shows such signals on MRI. These findings are considered to be useful for differential diagnosis between FCOD and other lesions in the future.

In this case, epithelium and capsule were not detected from the pathology of FCOD. These findings consistent with past report (23). Generally, it is known that the juvenile tissue is slower in wash-out time of the contrast medium than the mature tissue $(24,25)$.

Difference in wash-out time of contrast medium between juvenile fibroblasts and mature fibroblasts is considered as the reason of postcontrast T1-weighted MRI show the FCOD with peripheral contrast-enhancement.

\section{Conclusions}

In this time, we experienced FCOD of osteolytic stage. Especially, they were very useful findings that FCOD of osteolytic stage showed cyst-like findings on CT and MRI.
These findings suggested that it is necessary to suspect FCOD of osteolytic stage, when multiple cystic diseases were found in the jaw of Asian women since middle age.

\section{References}

1. Grace P. Fibro-osseous lesions/mandible and maxilla. In: Koenig LJ, Tamimi D, Petrikowski CG, Harnsberger HR, Ruprecht A, Benson BW, Van Dis M, Hatcher D, editors. Diagnostic imaging oral and maxillofacial $1^{\text {st }}$ ed.. Friesens: Amirsys; 2012. p. 80-83.

2. Daviet-Noual V, Ejeil AL, Gossiome C, Moreau N, Salmon B: Differentiating early stage florid osseous dysplasia from periapical endodontic lesions: a radiological-based diagnostic algorithm. BMC Oral Health, 17: 1-8, 2017.

3. Wright JM, Vered M: Update from the 4th edition of the world health organization classification of head and neck tumours: Odontogenic and maxillofacial bone tumors. Head Neck Pathol, 11: 68-77, 2017

4. Brannon RB, Fowler CB: Benign fibro-osseous lesions: a review of current concepts. Adv Anat Pathol, 8: 126-143, 
2001.

5. Sadda RS, Phelan J: Dental management of florid cementoosseous dysplosia. N Y State Dent J, 80: 24-26, 2014.

6. Sarmento DJ, Monteiro BV, de Medeiros AM, da Silveira EJ: Severe florid cemento-osseous dysplasia: a case report treated conservatively and literature review. Oral Maxillofac Surg, 17: 43-46, 2013.

7. Fenerty S, Shaw W, Verma R, Syed A, Kuklani R, Yang J, Ali S: Florid cemento-osseous dysplasia: review of an uncommon fibro-osseous lesion of the jaw with important clinical implications. Skeletal Radiol, 46: 581-590, 2017.

8. Eskandarloo A, Yousefi F: CBCT findings of periapical cemento-osseous dysplasia: a case report. Imaging Sci Dent, 43: 215-218, 2013.

9. Min CK, Koh KJ, Kim KA: Recurrent symptomatic cementoosseous dysplasia: A case report. Imaging Sci Dent, 48: 131-137, 2018.

10. Cavalcante MB, de Oliveira Lima AL, Júnior MA, Santos MB: Florid cemento-osseous dysplasia simultaneous the chronic suppurative osteomyelitis in mandible. J Craniofac Surg, 27: 2173-2176, 2016.

11. Delai D, Bernardi A, Felippe GS, da Silveira Teixeira C, Felippe WT, Santos Felippe MC: Florid cemento-osseous dysplasia: a case of misdiagnosis. J Endod, 41: 1923-1926, 2015.

12. Köse TE, Köse OD, Karabas HC, Erdem TL, Ozcan I: Findings of florid cemento- osseous dysplasia: a report of three cases. J Oral Maxillofac Res, 4: 4, 2013.

13. Bhandari R, Sandhu SV, Bansal H, Behl R, Bhullar RK: Focal cemento-osseous dysplasia masquerading as a residual cyst. Contemp Clin Dent, 3: 60-62, 2012.

14. Zegarelli EV, Kutscher AH, Napoli N, lurono F, Hoffman P: The cementoma-a study with 230 patients with 435 cementomas. Oral Surg Oral Med Oral Pathol, 17: 219-224, 1964.

15. Huh JK, Shin SJ: Misdiagnosis of florid cemento-osseous dysplasia leading to unnecessary root canal treatment: a case report. Restor Dent Endod, 38: 160-166, 2013.

16. Aiuto R, Gucciardino F, Rapetti R, Siervo S, Bianch AE: Management of symptomatic florid cemento-osseous dyspla- sia: Literature review and a case report. J Clin Exp Dent, 10: 291-295, 2018.

17. Galgano C, Samson J, Küffer R, Lombardi T: Focal cementoosseous dysplasia involving a mandibular lateral incisor. Int Endod J, 36: 907-911, 2003.

18. Cavalcante AS, Sgarbi FC, Agapito Lda C, Roveroni LH, Brandao AA, Cabral LA: Florid cemento-osseous dysplasia: a report of three cases. Gen Dent. 56: 186-190, 2008.

19. Singer SR, Mupparapu M, Rinaggio J: Florid cemento-osseous dysplasia and chronic diffuse osteomyelitis: report of a simultaneous presentation and review of the literature. J Am Dent Assoc, 136: 927-931, 2005.

20. Perschbacher S. Neoplasm, benign, odontogenic/mandible and maxilla. In: Koenig LJ, Tamimi D, Petrikowski CG, Harnsberger HR, Ruprecht A, Benson BW, Van Dis M, Hatcher D, editors. Diagnostic imaging oral and maxillofacial $1^{\text {st }}$ ed.. Friesens: Amirsys; 2012. p. 130-131.

21. Sidorowicz W, Kubasiewicz-Ross P, Dominiak M: Familial cherubism: clinical and radiological features. Case report and review of the literature. Eur J Paediatr Dent, 19: 213-217, 2018.

22. Koening LJ. Tumor-like lesions/mandible and maxilla. In: Koenig LJ, Tamimi D, Petrikowski CG, Harnsberger HR, Ruprecht A, Benson BW, Van Dis M, Hatcher D, editors. Diagnostic imaging oral and maxillofacial $1^{\text {st }}$ ed.. Friesens: Amirsys; 2012. p. 192-195.

23. Chi AC. Fibro-osseous lesions of the jaws/Bone pathology. In: Neville BW, Damm DD, Allen CM, Bouquot JE, editors. Oral and maxillofacial pathology $3^{\text {rd }}$ ed.. St. Louis: Saunders; 2009. p. 641-645.

24. Achiama M, Andersen L, M.Klein, Løgager V, Chabanova E, Thomsen H, Rosenberg J: Differentiation between benign and malignant colon tumors using fast dynamic gadoliniumenhanced MR colonography; a feasibility study. European Journal of Radiology, 74: 45-50, 2010.

25. Stack JP, Redmond OM, Codd MB, Dervan P, Ennis JT: Breast disease: Tissue characterization with Gd-DTPA enhancement profiles. Radiology, 174: 491-494, 1990. 